\section{Prognostic value of anemia in terms of disability and mortality in hospitalized geriatric patients: results from the CRIME study}

\author{
Gloria Brombo, ${ }^{1}$ Marta Dianin, ${ }^{1}$ \\ Lara Bianchi, ${ }^{1}$ Andrea Corsonello, ${ }^{2}$ \\ Antonio Cherubini, ${ }^{3}$ Carmelinda Ruggiero, ${ }^{4}$ \\ Graziano Onder, ${ }^{5}$ Stefano Volpato ${ }^{1}$ \\ 'Department of Medical Sciences, \\ University of Ferrara; ${ }^{2}$ Unit of Geriatric \\ Pharmacoepidemiology, Research \\ Hospital of Cosenza, Italian National \\ Research Center on Aging (INRCA), \\ Cosenza; ${ }^{3}$ Department of Geriatrics, \\ Research Hospital of Ancona, Italian \\ National Research Center on Aging \\ (INRCA), Ancona; ${ }^{4}$ Unit of Geriatric, S. \\ Maria della Misericordia Hospital, \\ University of Perugia, Perugia; ${ }^{5}$ Research \\ Center on Aging, Catholic University of \\ Sacred Heart, Rome, Italy
}

\section{Abstract}

Anemia, a common concern in geriatric population, is associated with several negative outcomes. The aim of this study was to investigate the independent prognostic value of anemia in the year after hospital discharge, analyzing its association with disability and mortality in a sample of older hospitalized patients. We evaluated 896 in-hospital older patients enrolled in the CRiteria to Assess Appropriate Medication Use among Elderly Complex Patients (CRIME) study, with assessment of hemoglobin levels at hospital admission and with follow-up data. We analyzed the risk of developing disability (in patients without pre-existing disability in activities of the daily living) and the likelihood of death (in the entire sample) in the 12 months after discharge according to presence and degree of anemia (defined by sex-specific World Health Organization criteria). Mean age of study participants was $81.2 \pm 7.4$ years, $57.8 \%$ had prevalent anemia. In unadjusted analysis, anemia was strongly associated with functional status and survival. Nevertheless, the increased risk of disability and death was influenced by the coexistence of several clinical conditions associated with anemia. Indeed, using multivariate logistic regression analysis adjusted for potential confounders, the association with disability was strongly attenuated [severe anemia odds ratio (OR) 1.86, 95\% confidence interval (CI): 0.96-3.58, mild-moderate anemia OR $1.05,95 \%$ CI: $0.62-1.80]$ and the relationship with mortality was no longer significant [ severe anemia hazard ratio (HR) 1.13, 95\% CI: $0.73-1.75$, mild-moderate anemia HR 1.14, 95\% CI: 0.78-1.67]. In older hospitalized patients, anemia, despite not influencing mortality, might have a significant disabling effect. Anemia should not be considered as an inevitable epiphenomenon of aging but a condition able to worsen the quality of life.

\section{Introduction}

Anemia is defined by World Health Organization (WHO) criteria as hemoglobin (Hb) levels lower than $12 \mathrm{~g} / \mathrm{dL}$ in women and lower than $13 \mathrm{~g} / \mathrm{dL}$ in men. ${ }^{1}$ This condition is common in geriatric population (the WHO reported a worldwide prevalence of anemia in elderly of 23.9\%) but in literature there is a broad variability of data on its exact incidence and prevalence. According to a review published in 2004, the prevalence of anemia ranged from $2.9 \%$ to $61 \%$ in elderly men and from $3.3 \%$ to $41 \%$ in elderly women; several factors are involved in this variability, including the setting of the study, the health status of the population, and the criteria used to define anemia. ${ }^{2}$ Anemia, being often an epiphenomenon of other pathological conditions, is extremely common especially in hospitalized elderly patients, in which prevalence reaches about $50 \%{ }^{3}$

Among hospitalized subjects, anemia has been associated with physical disability. A cross-sectional study performed on geriatric hospitalized patients found anemia to be associated with an increased likelihood of disability in activities of the daily living (ADL) ${ }^{4}$ but, stratifying the analysis by comorbidity severity, the association between anemia and disability remained significant only in patients with the lowest comorbidity levels. ${ }^{5}$ Furthermore, in elderly with ADL disability at hospital admission, Maraldi et al. demonstrated that anemia was inversely associated with the likelihood of regaining independence during hospital stay and, among anemic patients, the level of improvement was greater in subjects with higher Hb values. ${ }^{3}$

Since anemia is associated with many negative outcomes, some authors investigated its potential association with mortality. Several studies, performed on community-dwelling elderly, found a significant association between $\mathrm{Hb}$ levels and mortality. ${ }^{6-9}$ In one of these studies, where an increased all-cause mortality was found among elderly with mild anemia [hazard ratio (HR) 1.86, 95\% confidence interval (CI): 1.34-2.53], the authors conducted also a subgroup analysis by anemia type. The mortality risk was significantly increased in subjects with mild anemia of
Correspondence: Gloria Brombo, Department of Medical Sciences, Section of Internal and Cardiopulmonary Medicine, University of Ferrara, via Aldo Moro 8, I-44124 Ferrara, Italy. Tel.: +39.0532.237017 - Fax: +39.0532 .239547 .

E-mail: g.brombo@gmail.com

Key words: Elderly; anemia; disability; mortality.

Funding: the CRIME study was funded by the Italian Ministry of Labor, Health and Social Policy (Bando Giovani Ricercatori 2007, Convenzione $N^{\circ} 4$ ). The sponsors played no part in the study design; collection, analysis, or interpretation of data; the writing of the report; or the decision to submit the article for publication. The protocol of this study was approved by Ethics Committees and written consent was obtained from all participants.

Contributions: SV, MD, study concept and design; GB, LB, acquisition of the data; GB, MD, SV, analysis and interpretation of the data; GB, SV, manuscript writing; $\mathrm{GB}, \mathrm{LB}, \mathrm{MD}, \mathrm{ACo}, \mathrm{ACh}, \mathrm{CR}$, G0, SV, critical revision of the article and approval of the final version to be published.

Conflict of interests: the authors declare no potential conflict of interests.

Received for publication: 7 February 2016.

Revision received: 9 March 2016.

Accepted for publication: 24 March 2016.

This work is licensed under a Creative Commons Attribution-NonCommercial 4.0 International License (CC BY-NC 4.0).

(C) Copyright G. Brombo et al., 2016

Licensee PAGEPress, Italy

Geriatric Care 2016; 2:5803

doi:10.4081/gc.2016.5803

chronic disease (HR 5.44, 95\% CI: 3.53-8.06), while significant results were not found for the other anemia categories. ${ }^{6}$

The aim of our study was to evaluate the prognostic value of anemia after hospitalization, analyzing the association of anemia with functional disability and mortality in a sample of hospitalized elderly patients highly heterogeneous in terms of clinical features. Moreover, we assessed whether the potential association with negative clinical events is independent of the concomitant presence of other significant prognostic factors.

\section{Materials and Methods}

\section{Study population and data collection}

We used data from 1123 subjects enrolled in the CRiteria to Assess Appropriate Medication Use among Elderly Complex Patients (CRIME) 
project, an observational study performed in geriatric and internal medicine acute care wards of 7 Italian hospitals (Gemelli Hospital, Catholic University of Sacred Heart of Rome; University of Perugia; University of Ferrara; National Institute of Hospitalization and Care INRCA situated in Ancona, Cosenza, Fermo and Rome). Methodology of the CRIME project has been described in detail elsewhere. ${ }^{10,11}$

Briefly, all patients consecutively admitted to participating wards during the enrollment period (June 2010 - May 2011) entered the study. Exclusion criteria were age $<65$ years old and unwillingness to take part to the study. Ethical approval for the study was obtained in all participating centers. Hospitalized patients were invited to participate to the study and were free to decline participation. Written consent was obtained with assurance of data confidentiality. Data collection was conducted through a dedicated questionnaire, filled in at hospital admission and at discharge by study researchers. Moreover, patients were evaluated 3, 6 and 12 months after discharge. Followup was performed through ambulatory visits (or telephone interview if patient's clinical condition did not allow the visit). During each follow-up, participants' information was obtained by interviewing themselves or their relatives. For dead patients, the date of death was provided by relatives.

For this study, we further excluded patients with missing $\mathrm{Hb}$ value (3.9\%) and/or those lost to follow-up (17.3\%). Therefore, the total number of subjects included in the present analysis was 896 .

\section{Hemoglobin evaluation}

$\mathrm{Hb}$ levels of study participants were assessed at hospital admission after a 12-h overnight fast. Assays were performed in the central laboratory of each clinical center using standardized methods. Each laboratory fulfilled the requirements of a standard quality control program, thus ensuring the reliability and validity of all biochemical measures.

Anemia was defined according to sex-specific $\mathrm{WHO}$ criteria: $\mathrm{Hb}<13 \mathrm{~g} / \mathrm{dL}$ in men and $\mathrm{Hb}$ $<12 \mathrm{~g} / \mathrm{dL}$ in women. ${ }^{1}$ We further divided anemic patients in two groups: mild/moderate anemia (if $\mathrm{Hb}<12$ and $\geq 10 \mathrm{~g} / \mathrm{dL}$ in women and $\mathrm{Hb}<13$ and $\geq 11 \mathrm{~g} / \mathrm{dL}$ in men) and severe anemia (if $\mathrm{Hb}<10 \mathrm{~g} / \mathrm{dL}$ in women and $\mathrm{Hb}<11 \mathrm{~g} / \mathrm{dL}$ in men).

\section{Outcomes definition}

Death occurring in the year after discharge was used as a measure of long-term mortality. After discharged, patients were reassessed at 3,6 , and 12 months through either ambulatory visits or telephone contacts. The day of discharge from hospital was considered as the follow-up starting point. During each follow-up, data on living status and, eventually, the date of death were collected. Time from discharge to last follow-up was considered as temporal function in our study. No information regarding causes of death was gathered.

Functional status was evaluated at baseline and during the follow-up using the participant's dependency in ADL, with higher values indicating higher level of dependency; ${ }^{4}$ incident ADL disability was defined as the need of assistance in at least one of the five ADL (bathing, dressing, toileting, transfer and feeding) during the follow-up among patients without ADL difficulty at baseline.

\section{Covariates}

Cognitive status was assessed using the mini mental state examination (MMSE) ${ }^{12}$ performed during hospitalization in clinical stable conditions preferably the day before discharge. To assess the presence of depressive symptoms we used the geriatric depression scale. ${ }^{13}$

Socio-demographic characteristics of the study population included age and gender. History of smoking and number of hospitalizations in the last year were ascertained from a baseline interview. Body mass index (BMI, $\mathrm{kg} / \mathrm{m}^{2}$ ) was calculated from subject's weight and height, measured using objective standard techniques. The prevalence of specific medical conditions was established using standardized criteria that combined information from baseline interview, medical records, physical examination and blood test results. Disease categories were: hypertension, coronary heart disease, heart failure, diabetes mellitus, dementia (categorized in non-Alzheimer's and Alzheimer's dementia), gastritis, peptic ulcer, renal failure and cancer. We also determined some biochemical parameters at discharge: albumin, creatinine and C-reactive protein (CRP).

\section{Statistical analysis}

Selected socio-demographic and clinical characteristics of the study population were compared according to presence and degree of anemia (no anemia, $\mathrm{n}=378$; mild-moderate anemia, $\mathrm{n}=343$; severe anemia, $\mathrm{n}=175$ ), using a $\chi^{2}$ test and ANOVA model for categorical and continuous variables, respectively. To calculate the risk of developing disability during followup, because of the lack of the precise date of the event, we estimated the odds ratio (OR) and the $95 \%$ CI using multivariable logistic regression models adjusted for potential confounders, according to severity of anemia. Regression models were adjusted for several variables that can potentially act as confounding factors on the association between anemia and the risk of disability (variables clinically significant, variables found to be associated in univariate analysis and variables able to change the coefficient of association between anemia and disability). All these factors were initially included in the model; afterword the model was simplified removing unnecessary variables using a backward selection technique. On this basis, we created the following models: model 1 unadjusted; model 2 adjusted for age and sex; model 3 adjusted for age, sex, dementia and cancer; model 4 adjusted for age, sex, dementia, cancer, albumin and degree of disability at discharge. This specific analysis was limited to patients without ADL disability at hospital admission $(n=499)$.

Then, we performed the survival analysis in the entire sample to analyze the association between anemia and mortality using KaplanMeier curves and the log-rank test. HR and 95\% CI for each anemia category were estimated by Cox proportional hazard regression adjusting for potential confounders, with the absence of anemia as the reference category. We adjusted the results as follows: model 1 unadjusted; model 2 adjusted for age, sex and number of hospitalization in the last year; model 3 as model 2 but adjusted also for heart failure, dementia, peptic ulcer/gastritis, renal failure and cancer; model 4 as model 3 but adjusted also for BMI and albumin. All analyses were performed using Stata 13.0 for Windows (Stata Corporation, College Station, TX, USA).

\section{Results}

Mean age of study participants was $81.2 \pm 7.4$ years and 492 (54.9\%) were female. At hospital admission anemia was present in $57.8 \%$ of study population.

Table 1 presents selected socio-demographic, functional and clinical characteristics of the study population according to presence and degree of anemia. Subjects with anemia were significantly older $(\mathrm{P}<0.001)$, had lower BMI $(\mathrm{P}<0.001)$ and greater number of hospitalizations in the last year $(\mathrm{P}<0.001)$. They also had a worst cognitive and functional performance with lower MMSE score $(\mathrm{P}<0.001)$ and higher disability in $\mathrm{ADL}(\mathrm{P}<0.001)$ compared to patients without anemia. As regards medical conditions, subjects with anemia had a significantly higher prevalence of heart failure $(\mathrm{P}<0.001), \quad$ non-Alzheimer's dementia $(\mathrm{P}=0.007)$, gastritis $(\mathrm{P}=0.041)$, peptic ulcer $(\mathrm{P}=0.001)$, renal failure $(\mathrm{P}<0.001)$ and cancer $(\mathrm{P}<0.001)$, but lower prevalence of hypertension $(\mathrm{P}=0.005)$. The presence of anemia was also significantly associated with lower values of albumin $(\mathrm{P}<0.001)$ and higher concentrations of creatinine $(\mathrm{P}<0.001)$ and $\mathrm{CRP}$ $(\mathrm{P}=0.035)$. There was no significant association between anemia and other participants' characteristics including gender and history of smoking.

Figure 1 displays the likelihood to remain independent, in relation to time and $\mathrm{Hb}$ values. In this part of the study we considered only 
patients without disability, so from an initial complete functional autonomy for all the patients, during the follow-up there was a reduction in the percentage of subjects who maintained their independence in ADL. This reduction, although present in all groups, was significantly proportional to severity of anemia; in fact it was particularly evident in

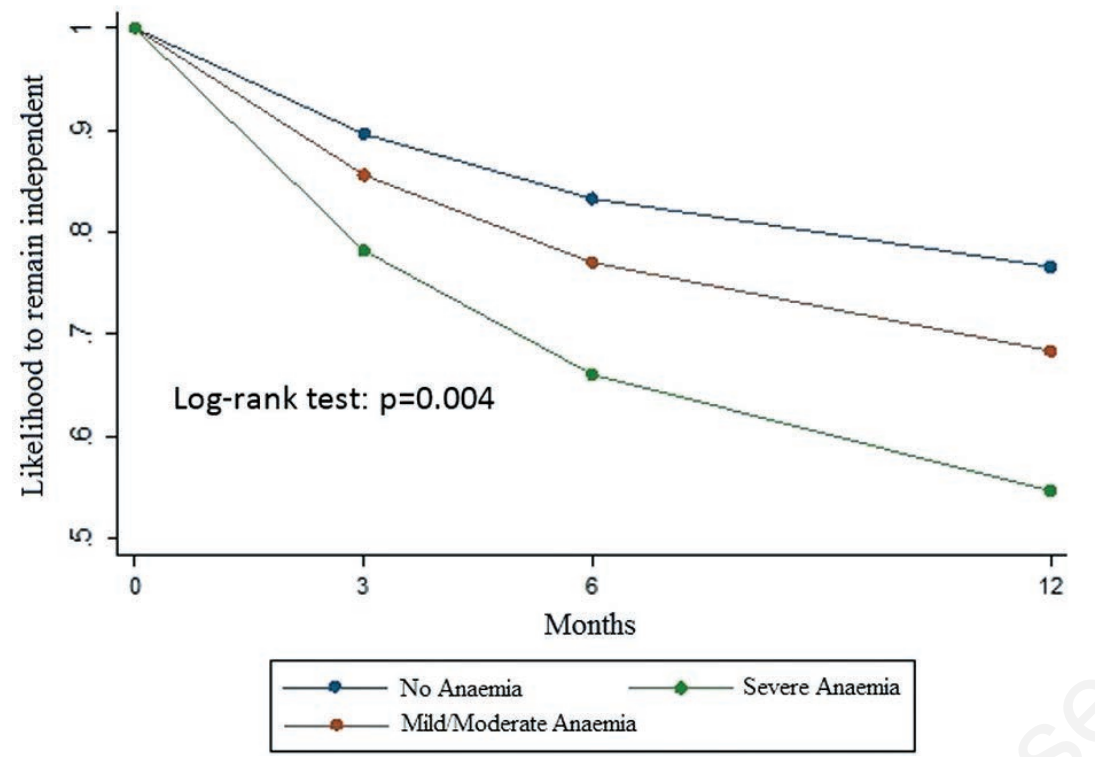

Figure 1. Likelihood to remain independent at 12 months in relation to anemia severity.

patients with severe anemia for which the likelihood of being still independent after 12 months was slightly higher than $50 \%$. We then analyzed the likelihood of developing disability according to degree of anemia, using a multivariate logistic regression analysis (Table 2). Model 1, unadjusted, shows that compared to patients without anemia, those with mild-moderate anemia had about one and a half fold higher risk of developing disability (OR 1.46, 95\% CI: $0.93-2.30$ ) and that subjects with severe anemia an almost three-fold increased risk (OR 2.72, 95\% CI: 1.53-4.83). Adjusting for several potential confounding variables, including functional status at discharge (complete independence or need of supervision), the risk of developing disability was similar in patients without anemia and with mild-moderate anemia (OR 1.05, 95\% CI: 0.62-1.80), while it is still higher, but at the limit of statistical significance, in subjects with severe anemia (OR 1.86, 95\% CI: 0.96-3.58).

Over 1 year of follow-up, 182 (20.3\%) participants had died. The 1-year survival of study participants, according to anemia severity, is shown in Figure 2. In the first 4 months of follow-up there was a greater survival in not anemic patients than in anemic ones, but no significant difference on the basis of anemia

Table 1. Selected characteristics of study participants according to presence and degree of anemia.

\begin{tabular}{|c|c|c|c|c|}
\hline Characteristics & $\begin{array}{l}\text { No anemia } \\
(\mathrm{n}=378)\end{array}$ & $\begin{array}{l}\text { Mild/moderate anemia } \\
\qquad(\mathrm{n}=343)\end{array}$ & $\begin{array}{l}\text { Severe anemia } \\
\quad(n=175)\end{array}$ & $\mathbf{P}$ \\
\hline Female, N (\%) & $200(52.9)$ & $199(58.0)$ & $93(53.1)$ & 0.338 \\
\hline Age, mean \pm SD & $78.8 \pm 7.4$ & $83.1 \pm 7.0$ & $82.7 \pm 6.7$ & $<0.001$ \\
\hline $\begin{array}{l}\text { Smoking, N (\%) } \\
\text { Former } \\
\text { Never-smoker }\end{array}$ & $\begin{array}{l}133(35.2) \\
205(54.2)\end{array}$ & $\begin{array}{l}112(32.7) \\
210(61.2)\end{array}$ & $\begin{array}{c}56(32.0) \\
107(61.1)\end{array}$ & 0.128 \\
\hline BMI $\left(\mathrm{kg} / \mathrm{m}^{2}\right)$, mean $\pm S D$ & $28.0 \pm 5.3$ & $25.8 \pm 5.0$ & $25.6 \pm 4.7$ & $<0.001$ \\
\hline $\begin{array}{l}\text { Hospitalizations in the last year, N (\%) } \\
\text { One } \\
\text { More than one }\end{array}$ & $\begin{array}{l}78(20.6) \\
62(16.4)\end{array}$ & $\begin{array}{l}77(22.4) \\
90(26.2)\end{array}$ & $\begin{array}{l}43(24.6) \\
53(30.3)\end{array}$ & $<0.001$ \\
\hline $\begin{array}{l}\text { Medical conditions, N (\%) } \\
\text { Hypertension } \\
\text { Coronary heart disease } \\
\text { Heart failure } \\
\text { Diabetes mellitus } \\
\text { Non-Alzheimer's dementia } \\
\text { Alzheimer's dementia } \\
\text { Gastritis } \\
\text { Peptic ulcer } \\
\text { Renal failure } \\
\text { Cancer } \\
\end{array}$ & $\begin{array}{c}303(80.2) \\
102(27.0) \\
74(19.6) \\
99(26.2) \\
38(10.0) \\
17(4.5) \\
34(9.0) \\
12(3.2) \\
63(16.7) \\
31(8.2)\end{array}$ & $\begin{array}{c}240(70.0) \\
116(33.8) \\
116(33.8) \\
100(29.2) \\
53(15.4) \\
24(7.0) \\
48(14.0) \\
8(2.3) \\
99(28.9) \\
48(14.0)\end{array}$ & $\begin{array}{c}127(72.6) \\
56(32.0) \\
65(37.1) \\
61(34.9) \\
34(19.4) \\
6(3.4) \\
27(15.4) \\
16(9.1) \\
64(36.6) \\
43(24.6)\end{array}$ & $\begin{array}{l}0.005 \\
0.125 \\
<0.001 \\
0.113 \\
0.007 \\
0.157 \\
0.041 \\
0.001 \\
<0.001 \\
<0.001\end{array}$ \\
\hline $\begin{array}{l}\text { Multidimensional assessment } \\
\text { MMSE, median (IQR) } \\
\text { GDS, median (IQR) } \\
\text { Disability in ADL, N (\%) }\end{array}$ & $\begin{array}{c}24(19,28) \\
3(2,6) \\
113(29.9)\end{array}$ & $\begin{array}{c}22(16,27) \\
4(2,7) \\
176(51.3)\end{array}$ & $\begin{array}{c}22(14,27) \\
4(3,7) \\
108(61.7)\end{array}$ & $\begin{array}{l}<0.001 \\
0.008 \\
<0.001\end{array}$ \\
\hline $\begin{array}{l}\text { Biochemical parameters (at discharge) } \\
\text { Albumin }(\mathrm{g} / \mathrm{dL}), \text { mean } \pm \text { SD } \\
\text { Creatinine }(\mathrm{mg} / \mathrm{dL}), \text { median }(\mathrm{IQR}) \\
\text { C-reactive protein }(\mathrm{mg} / \mathrm{L}) \text {, median }(\mathrm{IQR})\end{array}$ & $\begin{array}{c}3.8 \pm 0.5 \\
1.0(0.8,1.2) \\
3.7(1.1,10.3)\end{array}$ & $\begin{array}{c}3.5 \pm 0.6 \\
1.1(0.8,1.4) \\
6.3(2.3,17.9)\end{array}$ & $\begin{array}{c}3.4 \pm 0.7 \\
1.1(0.9,1.5) \\
6.0(1.5,23.9)\end{array}$ & $\begin{array}{c}<0.001 \\
<0.001 \\
0.035\end{array}$ \\
\hline
\end{tabular}

SD, standard deviation; BMI, body mass index; MMSE, mini mental state examination; IQR, interquartile range; GDS, geriatric depression scale; ADL, activities of daily living. 
severity was found. However, since the $4^{\text {th }}$ month the two curves started to space out, indicating a greater risk of death in patients with severe anemia than in those with a lower degree of anemia. The likelihood of 1-year mortality according to degree of anemia is further analyzed in Table 3 . The crude model shows that the risk of death was significantly more than doubled in patients with mild-moderate anemia (HR 2.30, 95\% CI: 1.60-3.31) and almost three-fold in subjects with severe anemia (HR 2.99, 95\% CI: 2.01-4.44) compared to not anemic patients. After adjustment for several confounders, the strength of the association between anemia and mortality progressively reduced. In fact, in the last model the relative risk was of $14 \%$ in mild-moderate anemic patients (HR 1.14, 95\% CI: 0.78-1.67) and of $13 \%$ in severe anemic ones (HR 1.13, 95\% CI: 0.73-1.75) and these differences did not reach significance.

\section{Discussion}

Our work demonstrated that the presence of anemia was associated with increased risk of developing disability in ADL and likelihood of death in the 12 months after discharge in a sample of older hospitalized patients. However, the increased risk of disability and particularly of death was strongly influenced and explained by the coexistence of several clinical conditions associated with the presence of anemia. In fact, from unadjusted data anemia seemed to have a strong impact on functional status and survival but, after adjustment for several potential confounders, the association with disability was no longer statistically significant and the relationship with mortality completely disappeared.

As previously mentioned, a populationbased study conducted by Riva et al. found mortality to be higher in patients with moderate anemia (defined as $\mathrm{Hb}$ concentrations between 10.0 and $11.9 \mathrm{~g} / \mathrm{dL}$ in women and between 10.0 and $12.9 \mathrm{~g} / \mathrm{dL}$ in men) compared to not anemic patients, despite the correction of results for many potential influential factors (such as age, gender, smoking, BMI, comorbidity, etc.). ${ }^{6}$ Another study, performed on community-dwelling elderly, found a significant $\mathrm{J}$ shaped relationship between $\mathrm{Hb}$ levels and mortality with lower and higher Hb concentrations and presence of anemia independently associated with increased mortality. ${ }^{7}$ Likewise, Culleton et al. found, always in a similar geriatric sample, $\mathrm{Hb}$ values to be associated with all-cause mortality in a J-shaped way, with the lowest mortality risk for $\mathrm{Hb}$ levels between 13.0

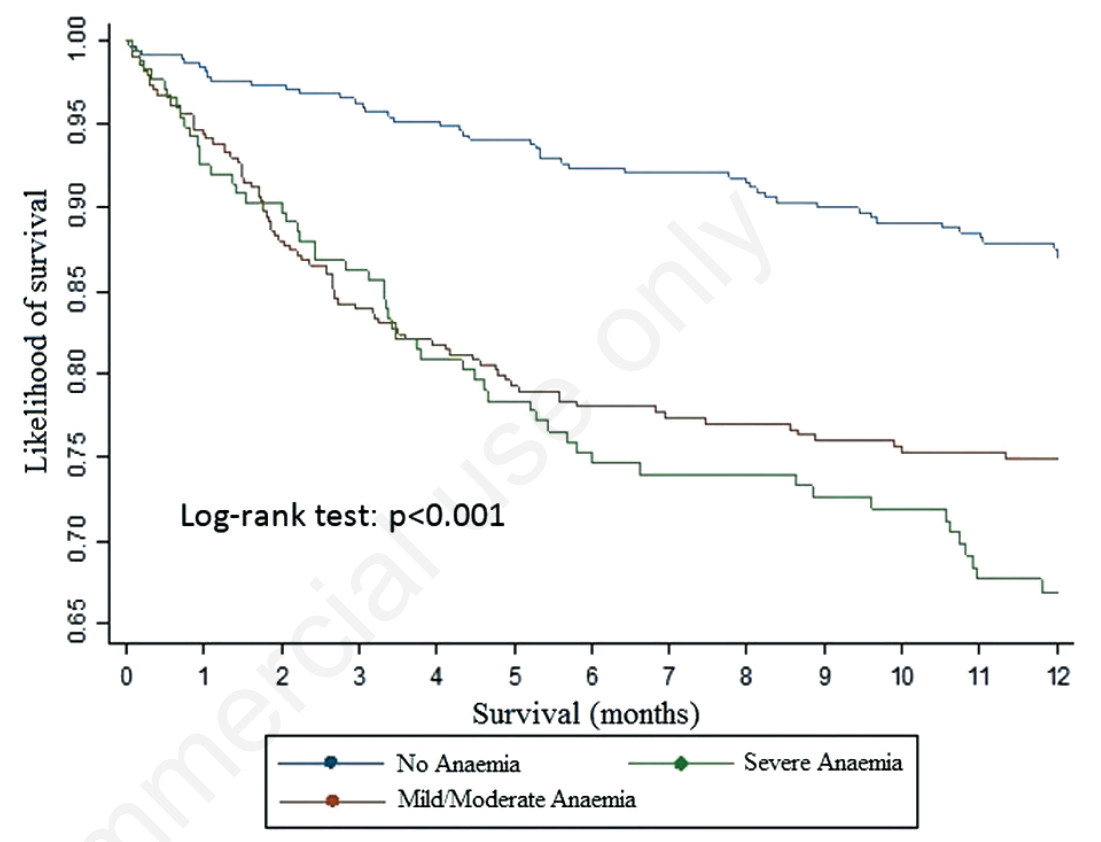

Figure 2. One-year survival of study participants, according to anemia severity.

Table 2. Crude and adjusted likelihood of disability according to degree of anemia.

\begin{tabular}{|c|c|c|c|c|c|c|}
\hline Anemia & $\mathbf{N}$ & $\begin{array}{c}\text { Disability } \\
(\%)\end{array}$ & $\begin{array}{c}\text { Model } 1 \\
\text { OR }(95 \% \text { CI })\end{array}$ & $\begin{array}{c}\text { Model } 2 \\
\text { OR }(95 \% \mathrm{CI})\end{array}$ & $\begin{array}{c}\text { Model } 3 \\
\text { OR }(95 \% \text { CI })\end{array}$ & $\begin{array}{c}\text { Model } 4 \\
\text { OR }(95 \% \mathrm{CI})\end{array}$ \\
\hline No anemia & 261 & 22.2 & 1 & 1 & 1 & 1 \\
\hline Mild/moderate & 153 & 29.4 & $1.46(0.93-2.30)$ & $1.13(0.70-1.83)$ & $1.13(0.69-1.85)$ & $1.05(0.62-1.80)$ \\
\hline Severe & 64 & 43.8 & $2.72(1.53-4.83)$ & $2.42(1.35-4.36)$ & $2.42(1.32-4.43)$ & $1.86(0.96-3.58)$ \\
\hline
\end{tabular}

Model 1: unadjusted; Model 2: adjusted for age and sex; Model 3: Model 2 + dementia (non-Alzheimer's and Alzheimer's) and cancer; Model 4: Model $3+$ albumin and degree of disability at discharge. OR, odds ratio; $\mathrm{Cl}$, confidence interval.

Table 3. Crude and adjusted likelihood of 1-year mortality according to degree of anemia.

\begin{tabular}{lccccccc} 
Anemia & N & Death & Model 1 & Model 2 & Model 3 & Model 4 \\
& & $(\%)$ & HR (95\% CI) & HR (95\% CI) & HR (95\% CI) & HR (95\% CI) \\
No anemia & 378 & 11.9 & 1 & 1 & 1 & 1 & 1 \\
Mild/moderate & 343 & 24.2 & $2.30(1.60-3.31)$ & $1.61(1.11-2.33)$ & $1.51(1.04-2.20)$ & $1.14(0.78-1.67)$ \\
\hline Severe & 175 & 30.9 & $2.99(2.01-4.44)$ & $2.06(1.38-3.09)$ & $1.71(1.13-2.59)$ & $1.13(0.73-1.75)$ \\
\hline
\end{tabular}

Model 1: unadjusted; Model 2: adjusted for age, sex and number of hospitalization in the last year; Model 3: Model $2+$ heart failure, dementia, peptic ulcer and/or gastritis, renal failure and cancer; Model 4: Model 3 + body mass index and albumin. $\mathrm{HR}$, hazard ratio; $\mathrm{Cl}$, confidence interval. 
and $15 \mathrm{~g} / \mathrm{dL}$ in women and 14.0 and $17.0 \mathrm{~g} / \mathrm{dL}$ in men. ${ }^{8}$ Finally, a study published in 2009 , performed on not hospitalized elderly patients without severe comorbidities, found an increased 5-year mortality risk in men with mild anemia (HR 1.9, 95\% CI: 1.5-2.4), while in women there was not a significant association between anemia and mortality risk. ${ }^{9}$ Our work led to different results compared to those of above studies since it showed that increased mortality would be present only without considering all the important prognostic factors associated with anemia in our sample. A possible explanation for these contrasting results is the different study population, consisted of hospitalized patients in our study instead of community-dwelling subjects of the others.

Regarding the development of functional decline, the relationship between anemia and disability has been already analyzed by other authors, who highlighted the role of comorbidity in determining the level of this association. Indeed, they found that much of this relationship was due to comorbidity presence, which is able to worsen any kind of clinical condition. ${ }^{5}$ From our results, we came to a similar conclusion: the risk of deterioration of functional status appeared the same in subjects without anemia and with mild-moderate anemia, but this risk tended to almost double in patients with severe anemia. The conclusions of our study are only partially in agreement with those obtained by other researchers: they are closer to the results of studies performed on hospitalized geriatric population, while they are discordant from those obtained from studies on elderly recruited from a territorial setting. Indeed, a study on community-dwelling women found a significant association between $\mathrm{Hb}$ concentrations and physical performance: women with higher Hb levels had a significant lower prevalence of mobility difficulty than those with lower Hb values, also in subjects with $\mathrm{Hb}$ levels within the normal range according to $\mathrm{WHO}$ criteria. ${ }^{14}$

One of the most important determining factors of the increased likelihood of development of disability in anemic patients, compared to subjects without anemia, seems to be the reduced tissue oxygenation. Indeed, the reduced availability of oxygen in the muscle causes a slowdown of metabolic pathways for energy production resulting in asthenia, fatigue, susceptibility to falls and fractures and consequences on degree of functional autonomy. These observations are supported also by a study published in 2004 in which low Hb levels were correlated with lower muscle strength, density and mass. ${ }^{15}$ Furthermore, the vitamin B12 deficiency, a possible cause of anemia and a common condition in elderly, can lead to neurological disorders such as dizziness or proprioceptive alterations that can predispose to falls, which are important causes of morbidity and mortality in elderly. ${ }^{16}$

Analyzing the results of this study, some limitations should be considered. The main one is that our control group cannot be considered healthy because it consists of elderly hospitalized patients, often disabled. Therefore, our patients are subjects with a condition of intrinsic fragility, independently of their $\mathrm{Hb}$ values. These particular conditions of the sample can certainly be considered a limitation, but also a merit of the study since this kind of population is rarely adequately analyzed. A second limitation is the low number of patients without disabilities at discharge that reduces the statistical power of the results. Finally, we did not have further information on anemia features (type, etiology, onset, micronutrient status related to iron profile or B-group vitamins). Indeed, in elderly patients there are several categories of anemia, including the unexplained one, that reflect different comorbidities and biochemical characteristics, ${ }^{17}$ based on these important differences, probably, a more exhaustive diagnosis of the type of anemia could influence outcomes.

\section{Conclusions}

On the basis of our results anemia, despite not having a significant relationship with mortality, appears to have an important negative impact on the functional status of the elderly. So our study permits to identify, among hospitalized geriatric population, a group of patients at higher risk for functional deterioration in which, possibly, the treatment of anemia could enhance both clinical and economic outcomes. Therefore, in elderly patients, anemia should not be considered as an inevitable epiphenomenon of aging but as a condition able to worsen the quality of life.

\section{References}

1. Benoist B, McLean E, Egli I, Cogswell M. Worldwide prevalence of anaemia 19932005. Global Database on Anaemia. Geneva: World Health Organization; 2008. Available from: http://www.who.int/vmnis/anaemia/ prevalence/en/ Accessed: October 2015.

2. Beghé C, Wilson A, Ershler WB. Prevalence and outcomes of anemia in geriatrics: a systematic review of the literature. Am J Med 2004;116:3S-10S.

3. Maraldi C, Volpato S, Cesari M, et al. Anemia and recovery from disability in activities of daily living in hospitalized older persons. J Am Geriatr Soc 2006;54: 632-6.

4. Katz S, Ford AB, Moskowitz RW, et al.
Studies of illness in the aged. The index of ADL: A standardized measure of biological and psychosocial function. JAMA 1963;185: 914-9.

5. Maraldi C, Ble A, Zuliani G, et al. Association between anemia and physical disability in older patients: role of comorbidity. Aging Clin Exp Res 2006;18:485-92.

6. Riva E, Tettamanti M, Mosconi $P$, et al. Association of mild anemia with hospitalization and mortality in the elderly: the Health and Anemia population-based study. Haematologica 2009;94:22-8.

7. Zakai NA, Katz R, Hirsch C, et al. A prospective study of anemia status, hemoglobin concentration, and mortality in an elderly cohort: the Cardiovascular Health Study. Arch Intern Med 2005;165:2214-20.

8. Culleton BF, Manns BJ, Zhang J, et al. Impact of anemia on hospitalization and mortality in older adults. Blood 2006;107:3841-6.

9. Endres HG, Wedding U, Pittrow D, et al. Prevalence of anemia in elderly patients in primary care: impact on 5-year mortality risk and differences between men and women. Curr Med Res Opin 2009;25:1143-58.

10. Fusco D, Lattanzio F, Tosato $\mathrm{M}$, et al. Development of CRIteria to assess appropriate Medication use among Elderly complex patients (CRIME) project: rationale and methodology. Drugs Aging 2009;26:3-13.

11. Tosato M, Settanni S, Antocicco M, et al. Pattern of medication use among older inpatients in seven hospitals in Italy: results from the CRiteria to assess Appropriate Medication use among Elderly complex patients (CRIME) project. Curr Drug Saf 2013;8:98-103.

12. Folstein MF, Folstein SE, McHugh PR. Minimental state. A practical method for grading the cognitive state of patients for the clinician. J Psychiatr Res 1975;12:189-98.

13. Sheikh JI, Yesavage JA. Geriatric depression scale (GDS): recent evidence and development of a shorter version. Clin Gerontol 1986;5:165-73.

14. Chaves PH, Ashar B, Guralnik JM, Fried LP. Looking at the relationship between hemoglobin concentration and prevalent mobility difficulty in older women. Should the criteria currently used to define anemia in older people be reevaluated? J Am Geriatr Soc 2002;50:1257-64.

15. Cesari M, Penninx BW, Lauretani F, et al. Hemoglobin levels and skeletal muscle: results from the InCHIANTI study. J Gerontol A Biol Sci Med Sci 2004;59:249-54.

16. Ocampo Chaparro JM. Vitamin B12 deficit and development of geriatric syndromes. Colomb Med (Cali) 2013;44:42-5.

17. Ferrucci L, Guralnik JM, Bandinelli S, et al. Unexplained anaemia in older persons is characterised by low erythropoietin and low levels of pro-inflammatory markers. $\mathrm{Br}$ 